\title{
BREAST CANCER IN A 23-YEAR-OLD NURSING MOTHER - CASE REPORT
}

Kathryn Müllen Aparecida de Assis Cabral'1, Ana Clara Vieira Ferreira', Ana Flávia Souza Salles e Silva', Ana Luiza Machado Costa'

${ }^{1}$ Faculdade de Medicina de Barbacena FUNJOBE - Barbacena (MG), Brazil.

Introduction: Breast cancer is uncommon in women below 35 years of age, and the diagnosis can be delayed due to higher breast density and less attention during clinical examination. Survival in young women is worse than in individuals from an older age group, and treatment may have a greater physical and psychological impact on these patients. This relevant report describes the clinical case of a 23 -year-old nursing mother, non-smoker, and without a family history of cancer, who detected a nodule in the left breast during self-examination. She sought medical care after three months due to nodule increase and local pain, which was treated as mastitis. Without improvement, she visited the mastology department, which identified, during clinical examination, a $9 \mathrm{~cm}$, mobile, ill-defined nodule, and negative axillary nodes. Breast ultrasound found two spiculated, heterogeneous nodules, with ill-defined borders in the left upper outer quadrant. Core biopsy revealed an invasive breast carcinoma with luminal B immunophenotype. The patient underwent neoadjuvant chemotherapy with good response, lumpectomy, and sentinel lymph node biopsy. She has been receiving chemotherapy for subsequent radiotherapy and hormone therapy. Objectives: Since currently breast cancer has no proven preventive measure, this work aims at highlighting the importance of raising awareness about the possibility of cancer among young patients for early diagnosis and prompt treatment so as to increase the survival of diagnosed patients. Discussion: Breast cancer is the main death-related neoplasm in the Brazilian female population, representing a challenge for public health. The main risk factors are family history, advanced age, and reproductive characteristics. Studies show that the most common histological type in young individuals is invasive ductal and that this age group has peculiarities concerning risk factors, tumor biology, genetics, prognosis, and social and psychological impact. Classic symptoms, such as breast and axillary nodules and changes in the breast and nipple skin, indicate the need to seek medical help. Treatment is not distinct from that of older age groups, but the diagnostic difficulty warns about the importance of paying attention to any suspicious sign of this disease. Regarding prevalence, breast cancer in young people is rarer than in older individuals, but recurrence is higher. Clinical suspicion can be hindered by the greater breast density and the lack of disease screening among these patients. Conclusion: Early clinical suspicion based on detailed medical history and physical examination contributes to effective diagnosis and therapeutic approach, seeking a better quality of life and higher patient survival. 